\title{
2 Some Global Results for Asymptotically Simple Space-Times
}

\author{
Gregory J. Galloway \\ University of Miami, Coral Gables, FL 33124, USA
}

\begin{abstract}
A uniqueness theorem for Minkowski space and de Sitter space associated with the occurrence of null lines (inextendible globally achronal null geodesics) is presented. This result is obtained as a consequence of the null splitting theorem, which is also discussed.
\end{abstract}

\section{$2.1 \quad$ Introduction}

The aim of this paper is to present some rigidity results for asymptotically simple space-times. By a space-time we mean a smooth connected time oriented Lorentzian manifold $(M, g)$ of dimension $\geq 3$, having signature $(-+\cdots+)$. We use standard notation for causal theoretic notions, e.g., for $A \subset M, I^{+}(A, M)$, the time-like future of $A$ in $M$ (resp., $I^{-}(A, M)$, the time-like past of $A$ in $\left.M\right)$, is the set of all points that can be reached from $A$ by a future directed (resp. past directed) time-like curve in $M$. For other basic definitions and results in spacetime geometry and causal theory we refer the reader to the standard references $[14,19]$.

Penrose's treatment of infinity $[18,20]$ in asymptotically flat space-times (and space-times with other asymptotic structures, as well) is based on his notion of asymptotic simplicity. A 4-dimensional, chronological space-time $(M, g)$ is asymptotically simple provided there exists a smooth space-time-with-boundary $(\tilde{M}, \tilde{g})$ such that,

(a) $M$ is the interior of $\tilde{M}$, and hence, $\tilde{M}=M \cup \mathscr{I}$, where $\mathscr{I}=\partial \tilde{M}$,

(b) $\tilde{g}=\Omega^{2} g$, where $\Omega$ is a smooth function on $\tilde{M}$ such that (i) $\Omega>0$ on $M$ and

(ii) $\Omega=0$ and $d \Omega \neq 0$ along $\mathscr{I}$, and

(c) every inextendible null geodesic in $M$ has a past and future end-point on $\mathscr{I}$.

Condition (c) is a strong global assumption, which implies that $M$ is null geodesically complete, and ensures that $\mathscr{I}$ includes all of null infinity. But it also rules out space-times with singularities, and black holes, etc. To treat such cases, condition (c) must be suitably weakened, cf. [3, 14, 22].

In this paper, we will be primarily interested in asymptotically simple spacetimes which obey the vacuum Einstein equation with cosmological term,

$$
\text { Ric }=\lambda g .
$$

If $\lambda=0$ (the asymptotically flat case) then $\mathscr{I}$ is necessarily a smooth null hypersurface, which decomposes into two parts, $\mathscr{I}^{+}$, future null infinity, and 
$\mathscr{I}^{-}$, past null infinity. If $\lambda>0$ (the asymptotically de Sitter case) then we have a similar decomposition, except that $\mathscr{I}$ is space-like.

We present here a uniqueness theorem for Minkowski space and for de Sitter space associated with the occurrence of null lines. A null line in a space-time $(M, g)$ is an inextendible null geodesic which is globally achronal (meaning that no two points can be joined by a time-like curve). Arguments involving null lines have arisen in many situations, such as the Hawking-Penrose singularity theorems [14], results on topological censorship [4,11], the Penrose-Sorkin-Woolgar approach to the positive mass theorem [21,23] (and related results on gravitational time delay [13]), and most recently results concerning the AdS/CFT Correspondence [12].

Every null geodesic in Minkowski space and de Sitter space is a null line. At the same time, each of these space-times, and indeed any space-time satisfying equation (2.1) (regardless of the sign of $\lambda$ ) obeys the null energy condition, $\operatorname{Ric}(X, X)=R_{i j} X^{i} X^{j} \geq 0$, for all null vectors $X$. In general, it is difficult for complete null lines to exist in space-times which obey the null energy condition. The null energy condition tends to focus congruences of null geodesics, which can lead to the occurrence of null conjugate points. A null geodesic containing a pair of conjugate points cannot be achronal. Thus we expect a space-time which satisfies the null energy condition and which contains a complete null line to be special in some way. The following theorem supports this point of view.

Theorem 2.1. Suppose $M$ is an asymptotically simple space-time satisfying the vacuum Einstein equation (2.1) with $\lambda \geq 0$. If $M$ contains a null line then $M$ is isometric to Minkowski space (if $\lambda=0$ ) or de Sitter space (if $\lambda>0$ ).

Let us give an interpretation of Theorem 2.1 in terms of the initial value problem for the vacuum Einstein equation, in the case $\lambda>0$. According to the fundamental work of Friedrich $[5,7]$, the set of asymptotically simple solutions to (2.1), with $\lambda>0$, is open in the set of all maximal globally hyperbolic solutions with compact spatial sections. Thus, by Theorem 2.1, in conjunction with the work of Friedrich, a sufficiently small perturbation of the Cauchy data on a fixed Cauchy hypersurface in de Sitter space will in general destroy all the null lines of de Sitter space, i.e., the resulting space-time that develops from the perturbed Cauchy data will not contain any null lines. While one would expect many of the null lines to be destroyed, it is somewhat surprising that none of the null lines persist. One is tempted to draw a similar conclusion in the $\lambda=0$ case. However, the nonlinear stability of asymptotic simplicity in this case has not been established, and, indeed may not hold, cf., [8,9] for further discussion. In fact at present, Minkowski space is the only known asymptotically simple solution to $(2.1)$ with $\lambda=0$ (though evidence is mounting that there are solutions distinct from, but in a suitable sense, close to Minkowski space; by Theorem 2.1, such solutions would have no null lines). Finally, we note that an asymptotically simple and de Sitter space-time $M$, which does not contain any null lines, will not have any particle horizons, i.e., the "past null cones" $\partial J^{-}(p)$ will be compact for all $p \in M$ sufficiently close to $\mathscr{I}^{+}$. As all such null cones 
in de Sitter space are non-compact, this further serves to illustrate the delicate nature of the causal structure of de Sitter space (see also [13, Corollary 1]).

Theorem 2.1 is a consequence of the null splitting theorem obtained in [10]. This latter result establishes the rigidity in general of null geodesically complete space-times which obey the null energy condition. We discuss this result in the next section. In Sect. 2.3 we present the proof of Theorem 2.1. For results of related interest concerning the rigidity of asymptotically simple space-times, see, for example, $[6,15]$.

\subsection{The Null Splitting Theorem}

The statement of the null splitting theorem involves null hypersurfaces. We recall briefly some facts about the geometry of null hypersurfaces; see e.g., [10, 14, 19] for more detailed discussions from slightly varying points of view.

A smooth null hypersurface in a space-time $(M, g)$ is a smooth co-dimension one submanifold $S$ along which the Lorentz metric $g$ is degenerate. Hence, $S$ admits a smooth future directed null vector field $K$, which is unique up to a positive scale factor. As is well-known, the integral curves of $K$, when suitably parameterized, are null geodesics, and are referred to as the null generators of $S$.

The null expansion tensor (or null second fundamental form) $\Theta$ of $S$ measures the variations in the spatial separation of the null generators of $S$. Let $T S / K$ denote the tangent vectors to $S \bmod K$; for each $p \in S, T_{p} S / K=\{\bar{X}: X \in$ $\left.T_{p} S\right\}$, where $\bar{X}=\left\{Y \in T_{p} S: Y=X \bmod K\right\} . T S / K$ is an $n-2$ dimensional vector bundle over $S(n=\operatorname{dim} M)$. For $\bar{X}, \bar{Y} \in T_{p} S / K$, define $h(\bar{X}, \bar{Y})=$ $g(X, Y) ; h$ is a well-defined smooth Riemannian metric on $T S / K$. $\Theta$ is defined as, $\Theta(\bar{X}, \bar{Y})=g\left(\nabla_{X} K, Y\right)$, where $\nabla$ is the Levi-Civita connection of $(M, g) ; \Theta$ is a well-defined symmetric bilinear form on $T S / K$, unique up to the scaling of $K$. By tracing $\Theta$ with respect to $h$, we obtain the null expansion scalar $\theta=h^{a b} \theta_{a b}$, which measures the divergence of the null generators towards the future. Along an affinely parameterized null generator, $s \rightarrow \eta(s)$, the null expansion $\theta=\theta(s)$ satisfies (provided $K$ is appropriately scaled) the Raychaudhuri equation for an irrotational null geodesic congruence,

$$
\frac{d \theta}{d s}=-\operatorname{Ric}\left(\eta^{\prime}, \eta^{\prime}\right)-\sigma^{2}-\frac{1}{n-2} \theta^{2},
$$

where $\sigma^{2}=\sigma_{a b} \sigma^{a b}$, and $\sigma_{a b}$ is the shear tensor, $\sigma_{a b}=\theta_{a b}-\frac{1}{n-2} \theta$.

We say that $S$ is totally geodesic if and only if the expansion tensor vanishes, $\theta_{a b} \equiv 0$, or, equivalently, if and only if the expansion scalar and shear vanish, $\theta \equiv 0, \sigma_{a b} \equiv 0$. This has the usual geometric meaning: A geodesic in $M$ starting tangent to a totally geodesic null hypersurface $S$ remains in $S$. Null hyperplanes in Minkowski space are totally geodesic, as is the event horizon in Schwarzschild space-time.

We now state the null splitting theorem. 
Theorem 2.2. Let $M$ be a null geodesically complete space-time which obeys the null energy condition, $\operatorname{Ric}(X, X)=R_{i j} X^{i} X^{j} \geq 0$, for all null vectors $X$. If $M$ admits a null line $\eta$ then $\eta$ is contained in a smooth achronal edgeless totally geodesic null hypersurface $S$.

The simplest illustration of Theorem 2.2 is Minkowski space: Each null line $\ell$ in Minkowski space is contained in a unique null hyperplane $\Pi$.

We make some comments about the proof, which is based on a maximum principle for $C^{0}$ null hypersurfaces, see [10] for details. First, by way of motivation, note that the null plane $\Pi$ above can be realized as the limit of the future null cone $\partial I^{+}(x)$ as $x$ goes to past null infinity along the null line $\ell$. $\Pi$ can also be realized as the limit of the past null cone $\partial I^{-}(x)$ as $x$ goes to future null infinity along the null line $\ell$. In fact, one sees that $\Pi=\partial I^{+}(\ell)=\partial I^{-}(\ell)$.

Thus, in the setting of Theorem 2.2, consider the achronal boundaries $S_{+}=$ $\partial I^{+}(\eta)$ and $S_{-}=\partial I^{-}(\eta)$. By standard causal theoretic results [19], $S_{+}$and $S_{-}$ are achronal, edgeless, $C^{0}$ (but in general not smooth) hypersurfaces in $M$. Since $\eta$ is a achronal, it follows that $S_{+}$and $S_{-}$both contain $\eta$. For simplicity, assume $S_{+}$and $S_{-}$are connected (otherwise restrict attention to the component of each containing $\eta$ ). The proof then consists of showing that $S_{+}$and $S_{-}$agree and form a smooth totally geodesic null hypersurface. We give some indication as to how this works.

By further properties of achronal boundaries (see especially [19, Lemma $3.19]$ ), each point $p \in S_{-}$is the past end point of a null geodesic contained in $S_{-}$which is future inextendible in $M$, and hence future complete, i.e., $S_{-}$is a $C^{0}$ null hypersurface ruled by future complete null geodesics. Similarly, $S_{+}$is a $C^{0}$ null hypersurface ruled by past complete null geodesics. Now suppose $S_{+}$and $S_{-}$are actually smooth null hypersurfaces. Then by standard arguments, based on Raychaudhuri's equation (eq. (2.2)), $S_{-}$must have null expansion $\theta_{-} \geq 0$. Indeed, if $\theta_{-}<0$ at some point $p$ then the null generator through $p$ would encounter a null focal point in a finite affine parameter time to the future, forcing the generator to leave $S_{-}$, which is impossible. (This is the basis of the proof of the black hole area theorem.) Time-dually, $S_{+}$has null expansion $\theta_{+} \leq 0$. Now, let $q$ be a point on both $S_{+}$and $S_{-}$. By simple causal considerations one observes that in the vicinity of $q, S_{+}$lies to the future side of $S_{-}$. Using the inequalities $\theta_{+} \leq 0 \leq \theta_{-}$, and the fact that the null expansion scalar can be expressed as a second order quasi-linear elliptic operator acting on some function related to the graph of the null hypersurface, one can apply the strong maximum principle to conclude that $S_{+}$and $S_{-}$agree near $q$, c.f., [10, Theorem II.1]. Thus, the nonempty set $S_{-} \cap S_{+}$is both open and closed in $S_{-}$, and in $S_{+}$. Hence $S_{-}=S_{+}$, and this common null hypersurface, call it $S$, must have vanishing null expansion, $\theta \equiv 0$. Equation (2.2) then implies that the shear of the generators also vanishes, and hence $S$ is totally geodesic.

The principal difficulty in proving Theorem 2.2 is in showing that the arguments of the preceding paragraph extend to the $C^{0}$ setting. In fact it is possible to show that $S_{+}$and $S_{-}$satisfy, $\theta_{+} \leq 0 \leq \theta_{-}$, in a certain weak sense, namely, in the sense of smooth support null hypersurfaces. Then, as an application of the 
weak version of the strong maximum principle obtained in [2] (which includes a regularity assertion), it is again possible to show that $S_{+}$and $S_{-}$agree and form a smooth null hypersurface with vanishing null expansion scalar, cf., [10, Theorem III.4].

\section{$2.3 \quad$ Proof of Theorem 2.1}

A proof of Theorem 2.1 in the case $\lambda=0$ was given in [10]. Here we give a proof of Theorem 2.1, different in a number of respects, which accommodates the case $\lambda>0$. We also correct a mistake in the proof given in [10]. The proof made use of the erroneous assertion that $M^{-}=M \cup \mathscr{I}^{-}$is causally simple (i.e., that the sets of the form $J^{ \pm}\left(K, M^{-}\right)$are closed for all compact $\left.K \subset M^{-}\right)$. As pointed out in [16], without some further assumption on $\mathscr{I}$, this in principle need not hold. In the proof presented here we circumvent the use of causal simplicity.

The first, and main step in proving Theorem 2.1, is to show that $(M, g)$ has constant curvature. Let $\eta$ be the assumed null line in $M$, and let $S$ be the component of $\partial I^{+}(\eta, M)$ containing $\eta$. By Theorem 2.2, $S$ is a smooth totally geodesic null hypersurface in $M$. The null line $\eta$ acquires a past end point $p$ on $\mathscr{I}^{-}$and a future end point $q$ on $\mathscr{I}^{+}$. We examine the structure of $S$ near $p$. (A similar structure will hold near q.) For this purpose it is convenient to extend $M^{-}=M \cup \mathscr{I}^{-}$slightly beyond its boundary. Thus, without loss of generality, we may assume that $M^{-}$is contained in a space-time (without boundary) $P$ such that $\mathscr{I}^{-}$separates $P$. It follows that $\mathscr{I}^{-}$is a globally achronal null hypersurface in $P$.

Observe that $I^{+}(\eta, M)=I^{+}(p, P)$, from which it follows that $\partial I^{+}(\eta, M)=$ $\partial I^{+}(p, P) \cap M$ (where $\partial I^{+}(A, X)$ refers to the boundary in $X$ ). Asymptotic simplicity then implies that the generators of $\partial I^{+}(\eta, M)$ must have past end points on $\mathscr{I}^{-}$at $p$. (A generator $\gamma$ of $\partial I^{+}(p, P)$ starting in $M$ either extends to $p$, or else is past inextendible in $P$, while remaining in $\partial I^{+}(p, P)$. In the latter case, $\gamma$ meets $\mathscr{I}^{-}$transversely and enters $I^{-}\left(\mathscr{I}^{-}, P\right)$, violating the achronality of $\mathscr{I}^{-}$.) The generators of $S \subset \partial I^{+}(\eta, M)$ must coincide with those of $\partial I^{+}(\eta, M)$, and hence have past end points on $\mathscr{I}^{-}$at $p$, as well. Let $U$ be a convex normal neighborhood of $p$, and let $A$ be the "null cone" in $U$ generated by the future directed null geodesics in $U$ emanating from $p$. $A$ is a smooth null hypersurface in $U$, except for the conical singularity at $p$. We can choose a smooth space-like hypersurface in $U$, passing slightly to the future of $p$, which meets $A$ in a 2 -sphere $\Sigma$. By invariance of domain, and the fact that $S$ is closed in $M$, it is easily seen that the subset $\Sigma \cap S$ of $\Sigma \cap M$ is both open and closed in $\Sigma \cap M$. It follows that all the null geodesics forming $A$ correspond precisely to the generators of $S$, except, in the asymptotically flat case, for the generator $\gamma_{p}$ of $\mathscr{I}^{-}$with past end point $p$. Note in particular, this implies that $S=\partial I^{+}(\eta, M)$.

Now, set $N_{p}=S \cup \gamma_{p}$ in the asymptotically flat case, and $N_{p}=S \cup\{p\}$ in the asymptotically de Sitter case. From the above, $N_{p}$ is made up of all the future inextendible null geodesics in $P$ emanating from $p$. In the asymptotically de Sitter case, $\mathscr{I}^{-}$is space-like, so $\partial I^{+}(p, P)$ meets $\mathscr{I}^{-}$only at $p$. It follows 
from statements above that $N_{p}=\partial I^{+}(p, P)$. Suppose, in the asymptotically flat case, that $\partial I^{+}(p, P)$ meets $\mathscr{I}^{-}$at a point $x$, say. Then there exists a null geodesic $\gamma \subset \partial I^{+}(p, P)$ with future end point $x$ which either extends in the past to $p$ or is past inextendible in $M \cup \mathscr{I}^{-}$. In the latter case, Lemma 4.2 in [16] implies that $M \subset I^{+}(\gamma)$, from which it follows that $M \subset I^{+}(p, P)$. But this contradicts the achronality of $\eta$. It follows that $\partial I^{+}(p, P) \cap \mathscr{I}^{-}=\gamma_{p}$, and again we have $N_{p}=\partial I^{+}(p, P)$. Thus, $N_{p}$ is an achronal boundary, and hence is an achronal edgeless $C^{0}$ hypersurface in $P$. Moreover, since the future directed null geodesics in $P$ emanating from $p$ do not cross and, being achronal, do not have points conjugate to $p, N_{p} \backslash\{p\}$ is the diffeomorphic image under the exponential map exp : $\mathcal{O} \subset T_{p} P \rightarrow P$ of $\left(\Lambda_{p}^{+} \backslash\{0\}\right) \cap \mathcal{O}$, where $\Lambda_{p}^{+}$is the future null cone in $T_{p} P$ and $\mathcal{O}$ is the maximal open set on which exp is defined. Thus, apart from the conical singularity at $p, N_{p}$ is a smooth null hypersurface. We are justified in thinking of $N_{p}$ as the future null cone in $P$ at $p$.

The shear tensor $\sigma_{a b}$ of $S$ in the physical metric $g$ vanishes, and so, since the shear is a conformal invariant, the shear tensor $\tilde{\sigma}_{a b}$ of $N_{p} \backslash\{p\}$ vanishes in the unphysical metric $\tilde{g}$. Then by the well-known propagation equation for the shear tensor [14, Eq. 4.36], the components $\tilde{C}_{a 0 b 0}$ (with respect to an appropriately chosen frame in which $e_{0}$ is aligned along the generators) of the conformal tensor of $\tilde{g}$ vanish on $N_{p} \backslash\{p\}$. We can now invoke an argument of Friedrich [6] used in essentially the same situation to prove a cosmic no-hair theorem. By use of the regular conformal field equations, specifically the divergencelessness of the rescaled conformal tensor,

$$
\tilde{\nabla}_{i} d_{j k l}^{i}=0, \quad d_{j k l}^{i}=\Omega^{-1} \tilde{C}_{j k l}^{i},
$$

Friedrich shows that the full rescaled conformal tensor $d_{j k l}^{i}$ vanishes on the future domain of dependence $D^{+}\left(N_{p}, P\right)$ of $N_{p}$. Hence, the conformal tensor with respect to the physical metric vanishes on $D^{+}\left(N_{p}, P\right) \cap M$. Together with equation (2.2), this implies that $(M, g)$ has constant curvature on $D^{+}\left(N_{p}, P\right) \cap M$. In a precisely time-dual fashion $(M, g)$ has constant curvature on $D^{-}\left(N_{q}, Q\right) \cap M$, where $Q$ is an extension of $M \cup \mathscr{I}^{+}$analogous to $P$, and $N_{q}$ is the past null cone in $Q$ at $q$.

To conclude that $M$ is everywhere of constant curvature we need to show that $M \subset D^{+}\left(N_{p}, P\right) \cup D^{-}\left(N_{q}, Q\right)$. Since $S=\partial I^{+}(\eta, M)=\partial I^{-}(\eta, M), M$ can be expressed as the disjoint union,

$$
M=I^{-}(S, M) \cup S \cup I^{+}(S, M) .
$$

This decomposition follows from [19, Proposition 3.15], but can be easily shown directly as follows. Let $x \in M \backslash S$. There exists a curve $\sigma$ in $M$ (not necessarily causal) from $x$ to $y \in S$ that meets $S$ only at $y$. Either $\sigma \cap I^{-}(S, M) \neq \emptyset$ or $\sigma \cap I^{+}(S, M) \neq \emptyset$. If the latter holds and $\sigma$ is not contained in $I^{+}(S, M)$, then $\sigma$ meets $\partial I^{+}(S, M)$ at some point $z \notin S$. But since $I^{+}(S, M)=I^{+}\left(\partial I^{+}(\eta, M)\right)=$ $I^{+}(\eta, M), z \in \partial I^{+}(\eta, M)=S$, which is a contradiction. Hence, $\sigma \subset I^{+}(S, M)$, and so $x \in I^{+}(S, M)$. Similarly, $\sigma \cap I^{-}(S, M) \neq \emptyset$ leads to $x \in I^{-}(S, M)$, which establishes (2.3). 
We now show that each term in (2.3) is a subset of $D^{+}\left(N_{p}, P\right) \cup D^{-}\left(N_{q}, Q\right)$. Trivially, $S \subset N_{p} \subset D^{+}\left(N_{p}, P\right)$. Consider $I^{+}(S, M) \subset J^{+}\left(N_{p}, P\right) \cap M$. We claim that $J^{+}\left(N_{p}, P\right) \cap M \subset D^{+}\left(N_{p}, P\right) \cap M$. If not, then $H^{+}\left(N_{p}, P\right) \cap M \neq \emptyset$. Choose a point $x \in H^{+}\left(N_{p}, P\right) \cap M$, and let $\nu$ be a null generator of $H^{+}\left(N_{p}, P\right)$ with future end point $x$. Since $N_{p}$ is edgeless, $\nu$ remains in $H^{+}\left(N_{p}, P\right)$ as it is extended into the past. By asymptotic simplicity, $\nu$ must meet $\mathscr{I}^{-}$. In fact, $\nu$ must meet $\mathscr{I}^{-}$transversely (even in the asymptotically flat case, since $\nu$ starts in $M$ ) and then enters $I^{-}\left(\mathscr{I}^{-}, P\right)$. But this means that $\nu$ has left $J^{+}\left(N_{p}, P\right)$, which is a contradiction. Hence, $I^{+}(S, M) \subset D^{+}\left(N_{p}, P\right)$, and by the time-dual argument, $I^{-}(S, M) \subset D^{-}\left(N_{q}, Q\right)$.

Thus, $M$ is globally of constant curvature. By the uniqueness of simply connected Lorentzian space forms, Theorem 2.1 will follow once we show that $M$ is simply connected and geodesically complete. In the asymptotically flat case, it is shown in [16] that $M$ is simply connected (in fact, is homeomorphic to $\mathbb{R}^{4}$ ). Simple connectivity in the anti-de Sitter case may be established as follows. Each generator of $S$ has a past end point at $p$ and a future end point at $q$. By the structure of $S$ near $p$ and $q$ established above, it follows that $S \cup\{p, q\}$ is homeomorphic to the 3 -sphere. Perturbing $S \cup\{p, q\}$ slightly near $p$ and $q$, we obtain a smooth achronal 3-sphere $S_{0}$ in $M$. By an argument similar to one given above, one can show that $H^{+}\left(S_{0}, P\right) \cap M=H^{+}\left(S_{0}, Q\right) \cap M=\emptyset$, from which it follows that $S_{0}$ is a Cauchy surface for $M$. (Alternatively, it follows from Proposition 2.1 in [1] that $M$ is globally hyperbolic. Then, since $S_{0}$ is compact and achronal it must be a Cauchy surface.) Thus $M$ has topology $\mathbb{R} \times S_{0}$, and so is simply connected.

It remains to show that $M$ is geodesically complete. Being asymptotically simple, we know that $M$ is null geodesically complete, which is essential to the proof of full completeness. Let $\bar{M}$ denote Minkowski space in the case $\lambda=0$, and de Sitter space in the case $\lambda>0$. By standard results, for each $x \in M$ and $\bar{x} \in \bar{M}$, there is a neighborhood of $x$ isometric to a neighborhood of $\bar{x}$. Since $M$ is simply connected, these local isometries can be pieced together, by an analytic continuation type argument, to produce a local isometry $\phi: M \rightarrow \bar{M}$, see, for example, Theorem 8.17 in [17]. (The assumption in [17, Th. 8.17] that $M$ is complete is not needed to produce the local isometry; it is used only to conclude that $\phi$ is a covering map.)

Let $x$ be any point in $M$, and let $\bar{x}=\phi(x)$. Then, from the fact that $\phi$ is a local isometry and $M$ is null geodesically complete, it follows that any (broken) null geodesic segment in $\bar{M}$ starting at $\bar{x}$ can be lifted uniquely via $\phi$ to a (broken) null geodesic segment in $M$ starting at $x$. From this it follows that $\phi$ is onto: Fix $x \in M$ and let $\bar{x}=\phi(x)$. Let $\bar{\nu}$ be a broken null geodesic in $\bar{M}$ from $\bar{x}$ to any other point $\bar{y}$. Let $\nu$ be the lift of $\bar{\nu}$ starting at $x$, and let $y$ be its final end point; since $\nu$ covers $\bar{\nu}$, we must have $\phi(y)=\bar{y}$.

To establish geodesic completeness, it is sufficient to show that any unit speed time-like or space-like geodesic $\gamma:[0, a) \rightarrow M, t \rightarrow \gamma(t)$, continuously extends to $t=a$. Let $\bar{\gamma}=\phi \circ \gamma ; \bar{\gamma}$ can be extended to a complete geodesic in $\bar{M}$ which we still refer to as $\bar{\gamma}$. Fixing $u_{0} \in[0, a)$ sufficiently close to $a$, one easily 
constructs a $C^{0}$ homotopy of curves $\left\{\bar{\sigma}_{u}\right\}, u_{0} \leq u<a$, from $\bar{\gamma}(0)$ to $\bar{\gamma}(a)$, where, for each $u \in\left[u_{0}, a\right), \bar{\sigma}_{u}$ consists of the segment $\left.\bar{\gamma}\right|_{[0, u]}$, followed by a suitably chosen two-segment broken null geodesic $\bar{\nu}_{u}$ from $\bar{\gamma}(u)$ to $\bar{\gamma}(a)$, such that the length of $\bar{\nu}_{u}$ (in some background Riemannian metric) goes to zero as $u \rightarrow a$. The broken null segments $\bar{\nu}_{u}$ can be constructed from the two families of null geodesics foliating a totally geodesic time-like 2-surface containing $\left.\bar{\gamma}\right|_{\left[u_{0}, a\right]}$. By the nature of the lifting procedure, the homotopy $\left\{\bar{\sigma}_{u}\right\}, u_{0} \leq u<a$ can be lifted to a $C^{0}$ homotopy $\left\{\sigma_{u}\right\}, u_{0} \leq u<a$, where, for each $u, \sigma_{u}$ consists of the segment $\left.\gamma\right|_{[0, u]}$, followed by a two-segment broken geodesic $\nu_{u}$ covering $\bar{\nu}_{u}$. The curves $\sigma_{u}$ must have a common final end point $q$, say. Since the length of $\nu_{u}$ goes to zero (in the lifted Riemannian metric) as $u \rightarrow a$, it follows that $\gamma(u) \rightarrow q$ as $u \rightarrow a$, i.e., $\gamma$ is extendible to $q$. This completes the proof of Theorem 2.1.

\subsection{Concluding Remarks}

It is possible to formulate a version of Theorem 2.1 applicable to anti-de Sitter space. However, since in this case $\mathscr{I}$ is time-like, the characteristic initial value problem encountered in the proof of Theorem 2.1 would become an initialboundary value problem, and it would be necessary to impose boundary data (namely the vanishing of the conformal tensor) on $\mathscr{I}$. In this case one might expect to establish the uniqueness of anti-de Sitter space without the assumption of a null line.

We believe that one should be able to generalize Theorem 2.1 in certain directions. For example, in the asymptotically flat case, we expect that the vacuum assumption could be weakened to allow for the presence of matter. For instance, if we assume space-time $M$ satisfies the null energy condition, and is vacuum in a neighborhood of $\mathscr{I}$, then the proof of Theorem 2.1 implies the existence of open sets near scri which are flat. If the vacuum region is analytic then it follows that a neighborhood of scri is flat, and so $M$ should have vanishing mass. By a suitable version of the positive mass theorem, $M$ should be isometric to Minkowski space. Thus, we conjecture that, in the asymptotically flat case, the vacuum assumption can be replaced by the null energy condition and a requirement that the space-time Ricci tensor falls off at an appropriate rate on approach to $\mathscr{I}$. It may also be possible, in the asymptotically flat case, to prove a version of Theorem 2.1 for weakly asymptotically simple space-times, which allows for the occurrence of black holes. By imposing suitable conditions on the domain of outer communications $I^{-}\left(\mathscr{I}^{+}\right) \cap I^{+}\left(\mathscr{I}^{-}\right)$(e.g., that the DOC be globally hyperbolic) one might expect to be able to show that the DOC is flat. To accomplish this, one may be able to exploit the fact that the proof of the null splitting theorem does not actually require the full null completeness of space-time. As the discussion of Theorem (2.2) shows, if $\eta$ is the null line, it is sufficient to require that the generators of $\partial I^{-}(\eta)$ (respectively, $\partial I^{+}(\eta)$ ) be future (resp., past) complete.

We mention in closing that the null splitting theorem has recently been used [1] to obtain restrictions on the topology of asymptotically simple and de Sitter 
space-times obeying the null energy condition. It is shown that the Cauchy surfaces of such space-times must be compact and have finite fundamental group.

\section{Acknowledgments}

This paper is based on a talk given at the Workshop on the Conformal Structure of Space-Times, held in Tübingen, April 2-4, 2001. We wish to thank the organizers Jörg Frauendiener and Helmut Friedrich for their gracious hospitality and support. We also wish to thank Piotr Chruściel for comments on an earlier draft.

\section{References}

1. L. Andersson and G. J. Galloway, $d S / C F T$ and spacetime topology, preprint, hepth/0202161.

2. L. Andersson, G. J. Galloway, and R. Howard, A strong maximum principle for weak solutions of quasi-linear elliptic equations with applications to Lorentzian and Riemannian geometry, Comm. Pure Appl. Math. 51, 581-624 (1998).

3. P. T. Chruściel, E. Delay, G. J. Galloway, and R. Howard, Regularity of horizons and the area theorem, Ann. Henri Poincaré 2, 109-178 (2001).

4. J. L. Friedman, K. Schleich, and D. M. Witt, Topological censorship, Phys. Rev. Lett. 71, 1486-1489 (1993).

5. H. Friedrich, On the existence of n-geodesically complete or future complete solutions of Einstein's field equations with smooth asymptotic structure., Comm. Math. Phys. 107, 587-609 (1986).

6. H. Friedrich, Existence and structure of past asymptotically simple solutions of Einstein's field equations with positive cosmological constant, J. Geom. Phys. 3, 101-117 (1986).

7. H. Friedrich, On the global existence and the asymptotic behaviour of solutions to the Einstein-Maxwell-Yang-Mills equations, J. Diff. Geom. 3, 275-345 (1991).

8. H. Friedrich, Einstein's equation and conformal structure, in The Geometric universe: Science, geometry and the work of Roger Penrose, eds. S. A. Huggett et al., Oxford University Press, Oxford, 1996.

9. H. Friedrich, Einstein's equations and geometric asymptotics, in Gravitation and relativity: At the turn of the millennium. Proceedings of the GR-15 Conference at Pune, India, in June 1997, eds. N. Dadhich and J. Narlikar, IUCAA, Pune, India.

10. G. J. Galloway, Maximum Principles for null hypersurfaces and null splitting theorems, Ann. Henri Poincaré 1, 543-567 (2000).

11. G. Galloway, K. Schleich, D. Witt, and E. Woolgar, Topological Censorship and Higher Genus Black Holes, Phys. Rev. D 60, 104039 (1999).

12. G. J. Galloway, S. Surya and E. Woolgar, A uniqueness theorem for the AdS soliton, Phys. Rev. Lett. 88, 101102 (2002).

13. S. Gao and R. M. Wald, Theorems on gravitational time delay and related issues, Class. Quant. Grav. 17, 4999-5008 (2000).

14. S.W. Hawking and G.F.R. Ellis, The large scale structure of space-time, Cambridge University Press, Cambridge, 1973.

15. L. J. Mason, The asymptotic structure of algebraically special spacetimes, Class. Quantum Grav. 15, 1019-1030 (1998). 
16. R.P.A.C. Newman, The global structure of simple space-times, Commun. Math. Phys. 123, 17-52 (1989).

17. B. O'Neill, Semi-Riemannian geometry, Academic Press, New York, 1983.

18. R. Penrose, Zero rest-mass fields including gravitation: asymptotic behavior, Proc. Roy. Soc. Lond. A 284, 159-203 (1965).

19. R. Penrose, Techniques of differential topology in relativity, SIAM, Philadelphia, 1972, (Regional Conf. Series in Appl. Math., vol. 7).

20. R. Penrose and W. Rindler, Spinors and Spacetime, vol. 2, chapter 9, Cambridge University Press, Cambridge, 1986.

21. R. Penrose, R. D. Sorkin and E. Woolgar, A positive mass theorem based on the focusing and retardation of null geodesics, preprint, gr-qc/9301015.

22. R. M. Wald, General relativity, University of Chicago Press, Chicago, 1984.

23. E. Woolgar, The positivity of energy for asymptotically anti-de Sitter spacetimes, Class. Quant. Grav. 11, 1881-1900 (1994). 